\title{
Synergistic Effects of Nano-Sized Clinoptilolite and Nigella Sativa on Inflammatory and Hematological Factors in Rats with Type 2 Diabetes
}

\author{
Elahe Bazri ${ }^{1}$, Sirous Khorram ${ }^{2}$, Mehran Mesgari $^{3}$, Mohammad Asghari-jafarabadi ${ }^{4}$, Ali Tarighat-Esfanjani ${ }^{5} \&$ \\ Yalda Salari ${ }^{6}$ \\ ${ }^{1}$ Faculty of Nutrition and Food Sciences, Tabriz University of Medical Sciences, Tabriz, I. R. Iran \\ ${ }^{2}$ Research Institute for Applied Physics and Astronomy, Experimental Physics, Materials Physics, and Materials \\ Science, University of Tabriz, Tabriz, I. R. Iran \\ ${ }^{3}$ Drug Applied Research Center, Tabriz University of Medical Sciences, Tabriz, I. R. Iran \\ ${ }^{4}$ Nutrition Science, Nutrition Research Center, Faculty of Nutrition and Food Sciences, Tabriz University of \\ Medical Sciences, Tabriz, I. R. Iran \\ ${ }^{5}$ Nutrition Research Center, School of Nutrition and Food Sciences, Tabriz University of Medical Sciences, \\ Tabriz, I. R. Iran \\ ${ }^{6}$ School of Nutrition and Food Sciences, Tabriz University of Medical Sciences, Tabriz, I. R. Iran \\ Correspondence: Ali Tarighat-Esfanjani, Nutrition Research Center/Faculty of Nutrition and Food Sciences, \\ Tabriz University of Medical Sciences, Attar Nishabouri St., Tabriz, I. R. Iran. Tel: 0098-41-33357582. E-mail: \\ Tarighata@tbzmed.ac.ir
}

Received: November 10, 2017

Accepted: November 25, 2018

Online Published: January 21, 2019

doi:10.5539/jmbr.v9n1p7

URL: https://doi.org/10.5539/jmbr.v9n1p7

\begin{abstract}
The inflammatory efficacy of supplementation of natural nano-sized clinoptilolite (NCLN) and Nigella sativa (NS) was evaluated At the end of $7^{\text {th }}$ week in interleukin1 $\beta$ (IL1 $\beta$ ), interleukin10 (IL-10), interleukin6 (IL-6) and platelet (PLT), white blood cell (WBC), red blood cell (RBC), hemoglobin (HGB), hematocrit (HCT), mean corpuscular hemoglobin $(\mathrm{MCH})$, mean corpuscular hemoglobin concentration (MCHC), mean corpuscular volume (MCV). 42 rats were divided into two groups as diabetic and non-diabetic. Diabetic group divided into 4 subgroups as normal control (NC), NS $1 \%$ food, NCLN $2 \%$ /food, NS $1 \%$ /food + NCLN $2 \%$ /food and fed high fat diet (HFD) for 1 month, then injected $35 \mathrm{mg} / \mathrm{kg}$ BW STZ to induce type 2 diabetes (T2D). Our results showed IL-10 in NCLN and NCLN+NS groups were significantly higher NC group $(\mathrm{p}<0.05)$. IL-6 decreased in NS group in comparison with DC group. And PLT decreased significantly in NCLN group in comparison with other groups. Our data suggests NS and NCLN may have synergistic beneficial effects on increasing some of anti-inflammatory factors.
\end{abstract}

Keywords: Type 2 Diabetes Mellitus, Inflammation, Nigella Sativa, Zeolites, Rats

\section{Introduction}

Diabetes mellitus (DM), which includes a group of metabolic disease, defined by hyperglycemia which result from impairs in insulin secretion, insulin action, or both of them (Association, 2014) that is categorized in different kinds such as: type-1, type-2 (Association, 2015). According to world health organization (WHO) criteria the prevalence of glucose intolerance was $31.1 \%$ impaired glucose intolerance (IGT) and $7.5 \%$ diabetes. The high prevalence of diabetes was accounted for type 2 diabetes mellitus (T2DM) in the age band 40-69 years. T2DM as one of the main causes of morbidity and mortality that several pathogenic processes are involved in the development of it. Several studies support the theory that inflammation associates with T2DM (Ehses et al., 2009) and recent researches assert the inflammation plays an important role in the pathogenesis and development of T2DM (Donath \& Shoelson, 2011). Here data show Beta-cell from T2DM patient secrete a pro-inflammatory cytokines profile and a defect in the ability to produce anti-inflammatory cytokines such as interluekine-10 (IL-10). As a result, T2DM creates an imbalance between pro-inflammatory and anti-inflammatory profiles that increased pro-inflammatory factor interluekine-1 $\beta$ (IL-1 $\beta$ ), interluekine-6 (IL-6) or decreased anti-inflammatory (IL-10) levels of cytokines (Ouchi et al., 2011). Thus, anti-inflammatory therapies could place in the prevention and treatment of T2DM. 
In diabetic patients, the development of vascular complication is associated with endothelial dysfunction. Additionally, recent indication has been reported change in hematologic indices might contribute to abnormal glucose metabolism and diabetes by increasing insulin resistance and hepatic dysfunction. As Mather of fact increased hematocrits values and blood viscosity are proposed as a major risk factors in developing T2DM (Farhangi et al., 2016). Hyperglycemia is thought to have harmful effect on the blood vessels for example it is suggested that the increased platelet enhances vascular complication in diabetic patients. In recent years, several studies demonstrated the participation of platelets as one of the coagulation system elements involved in genesis of these events and supporting the idea that the presence of increased platelet count may be one of the factors involved on the development of diabetes complication. on the basis of such previous studies, some researchers investigated the effects of NS on hematological factors (Besses et al., 1999).

Recently the medicine from plants has been drawn increasing attention worldwide, due to their low toxicity and few side-effects. Accordingly, world health organization has recommended the use of traditional plant for diabetes treatment, which they consider as excellent candidates for oral therapy (Modak et al., 2007).

Nigella sativa (NS) commonly known as black seed or black cumin is from the botanical family of Ranunculaceae that has been traditionally used in folk medicine. As a natural remedy, recent investigations have represented anti-diabetic (Kaleem et al., 2006), oxidative, anti-inflammatory (Al-Ghamdi, 2001), anti-hypertensive (Zaoui et al., 2000), anti-bacterial, bronchodilator (Razavi \& Hosseinzadeh, 2014), carcinogenesis inhibitory, immunopotentiating, and analgesic antimicrobial activity of NS in-vitro and in-vivo. Many animal studies revealed that NS and thymoquinone (TQ), the most important (active) compound inhibit inflammatory cytokines (Kanter et al., 2006). The use of NS can favor the secretion of Th2, versus Th1, cytokines by solenocyte (Alshatwi, 2014). The secretion of pro-inflammatory mediators, IL-1, IL-6 and TNF- $\alpha$, is significantly suppressed by NS and indicating an anti-inflammatory effects in vitro (Stenvinkel et al., 2005). And also previous studies clearly showed that the effect of NS on hematological factors and hemostasis system. Researches support the use of NS and its derivatives can be cause hematological change in diabetic animals (Asgary et al., 2012).

There is a little evidence in assessing the beneficial effect on T2DM, but there is some contradictory effects on blood factors (Martin-Kleiner et al., 2001). The uncertainty of the NS's effective type, dose in rats with T2DM and the report of similar effects such as adjustment and boost the immune system (Mohanna \& Nys, 1999).

Observations of therapeutic effects of NS in diabetes require high doses. Meanwhile, the use of high-dose NS compound has harmful side effects. Zeolites synthetic crystalline aluminosilicates with ion exchanging properties have been used in environmental, industries, agricultural and biomedical technologies (Lyu et al., 2017) and also recent studies showed that beneficial effects of zeolite in medicine for instance researches reported that zeolite could decrease glucose level in animal (Yapislar et al., 2016) furthermore several animal studies demonstrated the effects of zeolite on the immune system and hematological factors (Jawahar et al., 2016). So it seems that the simultaneous use of two complementary, in addition will be synergy effects probably can be prevented side effects with a low dose of two complementary (Pavelic \& Hadzija, 2003). Concurrent supplementation of NCLN and NS may cause synergistic effects in improvement of immune system while preventing allergic reaction and other side effects on vital organs.

Considering our earlier promising evidence on the effectiveness of nano-sized CLN (NCLN) on BG in T1DM, lack of studies assessing the effect of NCLN on inflammatory and hematologic factors and the uncertainty of the effective type and dose of NS in T2DM, this study aimed to test synergistic effects of these two materials on immune function and hematologic factors in T2DM rats.

\section{Methods}

\subsection{Chemicals}

Streptozotocin (STZ, Sigma Chemicals, and St. Louis, MO, USA), Diethyl ether and other solvents and buffers (Merck, Germany C.) were used in this project.

\subsection{Animals}

In this study, a total of 44 adult male Wistar rats, including 36 tasted and 6 healthy ones (normal control=NC), at aged 6-5 months, weighing more than $250 \mathrm{~g}$ were purchased from laboratory animal breeding center of Tabriz University of Medical Sciences. Diabetic rats were divided into 4 subgroups, each of them including 9 rats, as follows: (1) received only NCLN diet, (2) received only NS diet, (3) received both NS and NCLN diet, and (4) received standard rat's diet (as diabetic control=DC). Rats were chosen according to the following defined inclusion criteria: same age (5-6 month), sex (male) and race (Wistar), fasting blood glucose (BG) more than 
$250 \mathrm{mg} / \mathrm{dl}$, and proven diabetic through OGTT after 12 hours fasting. Moreover, any rats which died before the end of the study (12 weeks) were excluded (15 rats). All animals fed well on standard ad-labitum and normal drinking water and kept to acclimate to conditions of temperature $\left(23-25^{\circ} \mathrm{C}\right)$ and humidity $(30 \%$ to $50 \%)$ and light (cycle dark/light 12 hours) 4-5 teeth in cages. At the end of the study (week $7^{\text {th }}$ ), fasting blood glucose (FBG), lipid profile, hematologic factors, oxidative stress status, inflammatory and bone health markers assessed through the last blood sampling $(5 \mathrm{~mL})$ of the animals. All animal ethic laws respected in regard with the maintenance and operation of these animals and ethical cod obtained from the ethics committee of Tabriz University of Medical Sciences.

\subsection{Induction of T2DM in Rats}

T2DM was induced by a month-long high-fat diet (HFD) (32\% of energy from fat, $48 \%$ carbohydrate, $20 \%$ protein) followed through intraperitoneal injection of $35 \mathrm{mg} / \mathrm{kg}$ body weight a single dose STZ in $0.1 \mathrm{M}$ sodium citrate buffer, $\mathrm{pH} 4.5$ carried out. One week after injection, the diagnosis of diabetes, blood glucose levels with blood sampling from the orbital sinus (1-2 drops) performed. Blood glucose levels determined via using an Accu-Chek glucometer (Roche, Germany) and rats with blood sugar levels $>250 \mathrm{mg} / \mathrm{dl}$ selected and studied as diabetic.

\subsection{Oral Glucose Tolerance Test (OGTT)}

An OGTT with 12 hours fasting was conducted to ensure T2DM induction. Then, a $20 \%$ glucose $(2 \mathrm{~g} / \mathrm{kg} \mathrm{BW})$ solution was given to the animals, containing $20 \%$ glucose $(2 \mathrm{~g} / \mathrm{kg} \mathrm{BW})$. Blood samples were obtained from the animal's tail to measure BG and insulin levels, after 0,30,60 and 120 minutes. After 7 weeks, at the end of treatment, HOMA-IR was used for estimating insulin resistance as follows: Plasma glucose $(\mathrm{mg} / \mathrm{dl}) \times$ fasting plasma insulin $(\mathrm{mu} / \mathrm{L})$ divided by 405 .

\subsection{Preparation of Therapeutic Diets}

The seeds of NS purchased from Tabriz city's local market, and zeolite from Tehran (Afrazand Co., Tehran, Iran) then powdered using mixer grinder. Glow-discharge plasma was used to convert micro particles of CLN to nanorods (in the Research Institute for Applied Physics and Astronomy at University of Tabriz). The amounts of prescriptive Nigella Sativa and Nano-zeolites to intervention groups were respectively $1,000 \mathrm{mg} / \mathrm{kg} \mathrm{BW}$ and $2 \%$ daily for 8 weeks by combining powders with the rats' food and pelleted. The exact amount of prescribed supplements was variable based on weekly weight changes.

\subsection{Collection of Blood Samples and Estimation of Biochemical Parameters}

Serum IL-10, IL-1 $\beta$ and IL-6 (biochemical markers of inflammation) concentrations were determined using an ELISA kit (East Biopharma Co, China). And also blood PLT level and WBC, RBC, HGB, HCT, MCV, MCH, $\mathrm{MCHC}$ concentrations determined using an automated hematology analyzer (Sysmex Co, Japan).

\subsection{Statistical Analysis}

To description of the data mean and SD was calculated for all inflammatory and hematological factors. For comparison of the level of the mentioned factors across the five groups Kruskal-Wallis test was used because the sample sizes were too small and the underlying distribution of the inflammatory and hematological factors were unknown. Whenever Kruskal-Wallis test was significant, Mann-Whitney test was used to find out which groups differed. P-values were adjusted based on Bonferroni correction to prevent multiple comparison bias. Data analysis conducted by using SPSS software version 16 and P-value less than 0.05 considered statistically significant.

\section{Results}

\subsection{Inflammatory Factors}

NS and NCLN supplementation effects on inflammatory markers at the $7^{\text {th }}$ weeks of treatment presented in Table 2. Rats with diabetes had not significantly higher mean levels of serum IL-6 $(323.3 \pm 43.3 \mathrm{pg} . / \mathrm{ml}$ vs. $304.3 \pm 52.4 \mathrm{pg} . / \mathrm{ml})$ in comparison with healthy subjects. NC group had significantly higher mean levels of serum IL-10 in comparison with DC group ( $\mathrm{p}=0.013)$. In addition, NCLN group had significantly higher values in comparison with $\mathrm{NC}$ group $(\mathrm{p}=0.043)$ and $\mathrm{NCLN}+\mathrm{NS}$ group had significantly higher values in comparison with $\mathrm{NC}$ group ( $\mathrm{p}=0.021$ ). It observed no significant decrees in serum levels of IL6 decrease in NS group compared with DC group (297.8 $\pm 21.2 \mathrm{pg} . / \mathrm{ml}$ vs. $323.3 \pm 43.3)$ and also However, no significant difference found in serum IL-1 $\beta$ levels among groups. Regarding factor IL- $1 \beta$, the research team concluded that it should be excluded from the analysis as the data obtained on this factor suffered from laboratory errors. 


\subsection{Hematological Factors}

Comparison serum levels of hematological factors in rats shown in Table 3. NC group had significantly higher mean levels of serum PLT in comparison with NCLN group ( $\mathrm{p}=0.001)$. NS group had significantly higher values in comparison with NCLN group ( $\mathrm{p}=0.001$ ), NCLN+NS group had significantly lower values in comparison with NCLN group ( $\mathrm{p}=0.001$ ), and NS group had significantly higher values in comparison with DC group $(\mathrm{p}=0.002)$. And also NCLN group had significantly lower mean levels of serum MCHC in comparison with NC group ( $\mathrm{p}=0.024)$ and NS group had significantly lower values in comparison with $\mathrm{NC}$ group $(\mathrm{p}=0.013)$. However, other parameters had no significant difference among groups.

Table 1. Elemental composition of nano-sized clinoptilolite

\begin{tabular}{llllllll}
\hline & \multicolumn{7}{c}{ Weight (\%) } \\
& $\mathrm{Na}$ & $\mathrm{Al}$ & $\mathrm{Si}$ & $\mathrm{K}$ & $\mathrm{Si} / \mathrm{Al}$ & $\mathrm{Na} / \mathrm{Al}$ & $\mathrm{K} / \mathrm{Al}$ \\
\hline NCLN & 8.86 & 4.81 & 44.27 & 10.94 & 8.88 & 4.52 & 3.23 \\
\hline
\end{tabular}

$\mathrm{NCLN}=$ nano-sized clinoptilolite; $\mathrm{Na}=$ Sodium; $\mathrm{Al}=$ Aluminium; $\mathrm{Si}=\mathrm{Silicon} ; \mathrm{K}=$ Potassium.

Table 2. Mean \pm SD of inflammatory factors levels in rats after interventions

\begin{tabular}{lllll}
\hline Groups & Treatment & IL-1 $\mathbf{\beta}(\mathbf{p g} / \mathbf{d L})$ & IL-6 (pg/dL) & IL-10 (pg/dL) \\
\hline $\mathbf{1}$ & DC $(\mathrm{n}=6)$ & $<0.001 \pm 0.001$ & $323.3 \pm 43.3$ & $201.2 \pm 17.3$ \\
$\mathbf{2}$ & NCLN+NS (n=5) & $1.2 \pm 2.8$ & $374 \pm 77.2$ & $234.6 \pm 86.5$ \\
$\mathbf{3}$ & NS (n=5) & $612.3 \pm 1364.4$ & $297.8 \pm 21.2$ & $187.5 \pm 15.8$ \\
$\mathbf{4}$ & NCLN (n=7) & $4.09 \pm 10.8$ & $330.1 \pm 64.9$ & $195.2 \pm 23.1$ \\
$\mathbf{5}$ & NC $(\mathrm{n}=6)$ & $0.000 \pm 0.0000$ & $304.3 \pm 52.4$ & $181.4 \pm 16.9$ \\
$\mathbf{6}$ & P value & & 0.235 & 0.039 \\
\hline
\end{tabular}

SD: Standard deviation; IL=Interleukin; DC=Diabetic control, NCLN=Nano-sized clinoptilolite, NS=Nigella. Sativa, $\mathrm{NC}=$ Normal control. ANOVA test $(* \mathrm{P}$ value $<0.05$ between group $)$ followed by Post-Hoc tests. ${ }^{* *} \mathrm{P}$ value $<0.05$

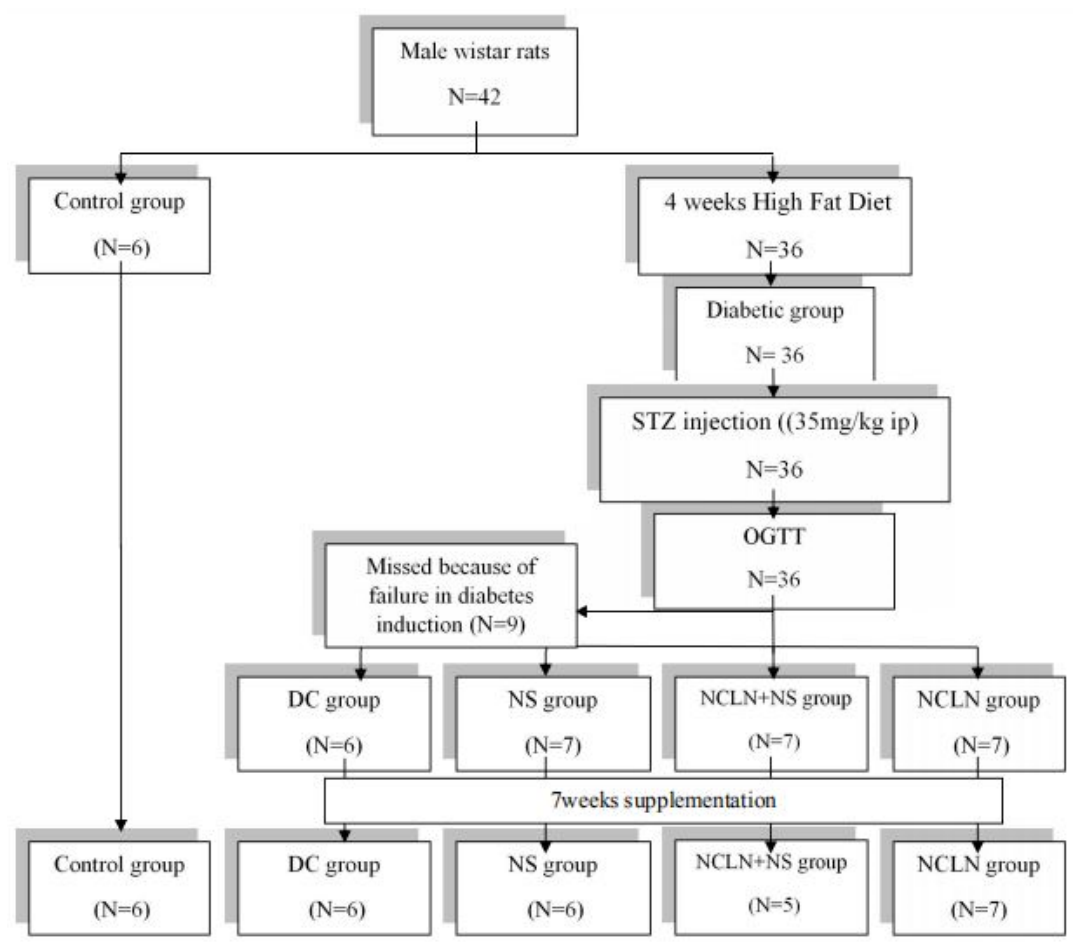

Figure 1. A diagram of study flow 
Table 3. Mean $\pm \mathrm{SD}$ of blood factors levels in rats after interventions

\begin{tabular}{llllllllll}
\hline Groups & Treatment & WBC & RBC & HGB & HCT & MCV & MCH & MCHC & PLT \\
\hline $\mathbf{1}$ & DC $(\mathrm{n}=6)$ & $10.1 \pm 4.9$ & $7.5 \pm 1.00$ & $13.4 \pm 0.96$ & $40.5 \pm 2.7$ & $54.0 \pm 5.4$ & $17.8 \pm 1.6$ & $33.1 \pm .6$ & $620.1 \pm 83.1$ \\
$\mathbf{2}$ & NCLN+NS $(\mathrm{n}=5)$ & $9.6 \pm 3.2$ & $7.7 \pm 0.97$ & $13.4 \pm 1.4$ & $40.5 \pm 3.8$ & $52.6 \pm 3.9$ & $17.4 \pm .61$ & $33.2 \pm 1.7$ & $646.2 \pm 117.1$ \\
$\mathbf{3}$ & NS (n=5) & $11.6 \pm 2.6$ & $6.8 \pm 1.5$ & $11.7 \pm 2.3$ & $36.2 \pm 5.8$ & $53.7 \pm 6.40$ & $17.1 \pm 1.2$ & $32.1 \pm 1.6$ & $645.3 \pm 134.5$ \\
$\mathbf{4}$ & NCLN (n=7) & $8.8 \pm 3.9$ & $7.2 \pm 0.78$ & $12.6 \pm 1.1$ & $39.1 \pm 2.8$ & $54.4 \pm 3.2$ & $17.5 \pm 0.61$ & $32.3 \pm 0.98$ & $449.2 \pm 108.7$ \\
$\mathbf{5}$ & NC (n=6) & $9.4 \pm 1.5$ & $7.9 \pm 0.4$ & $13.0 \pm 0.79$ & $38.4 \pm 2.2$ & $48.7 \pm 1.6$ & $16.5 \pm .44$ & $33.8 \pm 0.7$ & $658.1 \pm 44.7$ \\
$\mathbf{6}$ & P value & 0.635 & 0.375 & 0.138 & 0.168 & 0.184 & 0.202 & $0.048^{*}$ & $0.02 *$ \\
\hline
\end{tabular}

$\mathrm{DC}=$ Diabetic control, NCLN=Nano-sized clinoptilolite, NS=Nigella. Sativa, NC=Normal control, platelet (PLT), white blood cell (WBC), red blood cell (RBC), hemoglobin (HGB), hematocrit (HCT), mean corpuscular hemoglobin $(\mathrm{MCH})$, mean corpuscular hemoglobin concentration (MCHC), mean corpuscular volume (MCV). ANOVA test $(* \mathrm{P}$ value $<0.05$ between group $)$ followed by Post-Hoc tests. $* * \mathrm{P}$ value $<0.05$

\section{Discussions}

In this current study, T2DM induction made by high fat diet for 4 week, with a low dose STZ $(35 \mathrm{mg} / \mathrm{kg})$. This model reported to be similar to the T2DM in human (Gholamnezhad et al., 2016). This study evaluated whether the synergistic and separate of NS and NCLN supplementation could influence biomarker of inflammation hematological factors in diabetic rat. According to the evidence, this is the first study on the synergistic effects of supplementation of the NS and NCLN on inflammatory markers on diabetic rats.

Cytokines profile (IL10, IL6 and IL1 $\beta$ ) and hematological factors (WBC, RBC, HGB, HCT, MCV, MCH, MCHC and PLT) examined in diabetic rats. IL-10, an anti-inflammatory cytokine that normally released locally from immune cells, was significantly different between NS+NCLN group and DC group. The result indicated an increased anti-inflammatory factor IL10 in NS+NCLN group in comparison with DC group and it was significant $(\mathrm{P}<\ldots .$.$) and among other groups were not significant. Other inflammatory cytokines, IL-6 and IL-1 \beta$, were not significantly different among groups. On the other hand, the mean of IL6 in NS group decreased in comparison with NC and DC groups but was not significant. And also IL1 $\beta$ decreased in NS+NCLN group in comparison with NS and NCLN groups but there was not significant. The hematological factors were a part of this study. In current study, there has been a significant decrease in PLT in NS+NCLN group in comparison with NCLN and a significant decrease in NCLN in NC group in comparison with NCLN and also a significant decrease in NCLN group in comparison with NS. Serum MCHC levels mean were lower in the NCLN and NS group compared to healthy rats.

Our findings supported by other researches and the anti-inflammatory effects of NS reported in previous study. Lamprecht and et al. Demonstrated an increase in the anti-inflammatory IL-10 by a trend in the zeolite supplemented group which is compared to placebo (Lamprecht et al., 2015). Wu QJ and et al. showed significant decreases in the plasma concentration of TNF- $\alpha$, IL-1 $\beta$ and IL-6 (Wu et al., 2013).

But the results seem to be in conflict with previous findings regarding with pro-inflammatory cytokines (IL6 and IL1) and also in current experiment.

Such effects in this study were not significant on most of the biochemical parameters. Martin Houghton and et al., utilized NS crude fixed oil and pure thymoquinone to investigate inflammation pathway in rat peritoneal leukocytes. They revealed that both inhibited the cyclo-oxygenase and 5-lipoxygenase pathways of arachidonate metabolism in rat peritoneal leukocytes stimulated with calcium ionophore A23187. They indicated the protective usage of NS and its derived products for rheumatism and related inflammatory diseases (Houghton et al., 1995). In another investigation, the anti-inflammatory effects of NS seeds evaluated by Mutabagani and El-Mahdy. They suggested that the anti-inflammatory activity of NS seeds oil is related to inhibiting the generation of eicosanoids and lipid peroxidation (Mutabagani \& El-Mahdy, 1997). Valiollah Hajhashemi and et al obtained very similar findings using NS seeds oil in mice (Hajhashemi et al., 2004). Inhibition of inflammatory mediators (IL-1, IL-6), lypo-oxygenase and cyclo-oxygenase/Macrophage iNOS enzyme inhibition by extract thymoquinone and strengthen the immune system by increasing macrophage activity and lymphocyte numbers/Inhibition of inflammatory processes by thymoquinone/strengthening the immune response, especially in $\mathrm{T}$ cells by alpha linoleic acid, stearic acid and other NS seed compounds (Kooti et al., 2016). As shown by Zaoui et al. Treatment with NS was decreased platelet (Zaoui et al., 2002). According to Ahmad et al. platelet unchanged after NS oil treatment (Asgary et al., 2012). While our findings contradict the findings of other studies Al-Hader et al., demonstrated NS fixed oil significantly decreased platelet count an increased hematocrit and hemoglobin 
concentrations (Kooti et al., 2016) and Asgari et al., showed that significantly increased PLT count and decreased WBC count in rat (Asgary et al., 2012). But Al-Jishi did not find any change in blood cells when NS oil was given to normal rat (Asgary et al., 2012).

The shortcomings of this study have been clearly recognized. The researchers assessed an association between few inflammatory cytokines and small sample size. The main weakness in this paper seem to be relatively short time course between T2DM induction and start of intervention and lack of inflammatory profile measurements before intervention.

\section{Conclusion}

Supplementation with NS and NCLN was efficacious in increasing anti-inflammatory marker IL-10 but simultaneous supplementation of NS and NCLN had no synergistic effect on decreased pro-inflammatory factors IL-1 $\beta$ and IL-6. Although, the zeolite is widely used in industry, agriculture and environmental protection, their effects in appropriate animal models and possible medical applications are needed more studies. On the other hand, we can offer the zeolite alone or combination with other compounds because they can have a significant role in the increased anti-inflammatory markers. Our findings support the hypothesis that some specific inflammatory factors may play a role in the pathogenesis of T2DM and will offer new insights into the therapeutic targets for T2DM and related complication. Further research is needed to explore mechanistic explanation for the observations in this study.

\section{Acknowledgements}

This research study was financially supported by Tabriz University of Medical Sciences.

\section{Conflict of interests}

The authors declare that there is no conflict of interests regarding the publication of this paper.

\section{References}

Al-Ghamdi, M. (2001). The anti-inflammatory, analgesic and antipyretic activity of Nigella sativa. Journal of Ethnopharmacology, 76, 45-48.

Alshatwi, A. A. (2014). Bioactivity-guided identification to delineate the immunomodulatory effects of methanolic extract of Nigella sativa seed on human peripheral blood mononuclear cells. Chinese Journal of Integrative Medicine, 1-6.

Asgary, S., Najafi, S., Ghannadi, A., Dashti, G., \& Helalat, A. (2012). Efficiency of black cumin seeds on hematological factors in normal and hypercholesterolemic rabbits. ARYA Atherosclerosis, 7, 146.

Association, A. D. (2014). Diagnosis and classification of diabetes mellitus. Diabetes Care, 37, S81-S90.

Association, A. D. (2015). 2. Classification and diagnosis of diabetes. Diabetes Care, 38, S8-S16.

Besses, C., Cervantes, F., Pereira, A., Florensa, L., ..., \& Montserrat, E. (1999). Major vascular complications in essential thrombocythemia: A study of the predictive factors in a series of 148 patients. Leukemia, 13, 150-154.

Donath, M. Y., \& Shoelson, S. E. (2011). Type 2 diabetes as an inflammatory disease. Nature reviews. Immunology, 11, 98.

Ehses, J. A., Ellingsgaard, H., Böni-Schnetzler, M., \& Donath, M. Y. (2009). Pancreatic islet inflammation in type 2 diabetes: From $\alpha$ and $\beta$ cell compensation to dysfunction. Archives of Physiology and Biochemistry, 115, 240-247.

Farhangi, M. A., Javid, A. Z., \& Dehghan, P. (2016). The effect of enriched chicory inulin on liver enzymes, calcium homeostasis and hematological parameters in patients with type 2 diabetes mellitus: A randomized placebo-controlled trial. Primary Care Diabetes, 10, 265-271.

Gholamnezhad, Z., Havakhah, S., \& Boskabady, M. H. (2016). Preclinical and clinical effects of Nigella sativa and its constituent, thymoquinone: A review. Journal of Ethnopharmacology, 190, 372-386.

Hajhashemi, V., Ghannadi, A., \& Jafarabadi, H. (2004). Black cumin seed essential oil, as a potent analgesic and antiinflammatory drug. Phytotherapy Research, 18, 195-199.

Houghton, P. J., Zarka, R., de las Heras, B., \& Hoult, J. (1995). Fixed oil of Nigella sativa and derived thymoquinone inhibit eicosanoid generation in leukocytes and membrane lipid peroxidation. Planta Medica, 61, 33-36. 
Jawahar, S., Nafar, A., Vasanth, K., Musthafa, M. S., Arockiaraj, J., Balasundaram, C., \& Harikrishnan, R. (2016). Dietary supplementation of Zeolite on growth performance, immunological role, and disease resistance in Channa striatus against Aphanomyces invadans. Fish \& Shellfish Immunology, 51, 161-169.

Kaleem, M., Kirmani, D., Asif, M., Ahmed, Q., \& Bano, B. (2006). Biochemical effects of Nigella sativa L seeds in diabetic rats.

Kanter, M., Coskun, O., \& Uysal, H. (2006). The antioxidative and antihistaminic effect of Nigella sativa and its major constituent, thymoquinone on ethanol-induced gastric mucosal damage. Archives of Toxicology, 80, 217-224.

Kooti, W., Hasanzadeh-Noohi, Z., Sharafi-Ahvazi, N., Asadi-Samani, M., \& Ashtary-Larky, D. (2016). Phytochemistry, pharmacology, and therapeutic uses of black seed (Nigella sativa). Chinese Journal of Natural Medicines, 14, 732-745.

Lamprecht, M., Bogner, S., Steinbauer, K., Schuetz, B., ..., \& Wallner-Liebmann, S. (2015). Effects of zeolite supplementation on parameters of intestinal barrier integrity, inflammation, redoxbiology and performance in aerobically trained subjects. Journal of the International Society of Sports Nutrition, 12, 40.

Lyu, W., Jia, H., Deng, C., Saito, K., Yamada, S., \& Kato, H. (2017). Zeolite-Containing Mixture Supplementation Ameliorated Dextran Sodium Sulfate-Induced Colitis in Mice by Suppressing the Inflammatory Bowel Disease Pathway and Improving Apoptosis in Colon Mucosa. Nutrients, 9, 467.

Martin-Kleiner, I., Flegar-Meštrić, Z., Zadro, R., Breljak, D., ..., \& Boranić, M. (2001). The effect of the zeolite clinoptilolite on serum chemistry and hematopoiesis in mice. Food and Chemical Toxicology, 39, 717-727.

Modak, M., Dixit, P., Londhe, J., Ghaskadbi, S., \& Devasagayam, T. P. A. (2007). Recent Advances in Indian Herbal Drug Research Guest Editor: Thomas Paul Asir Devasagayam Indian Herbs and Herbal Drugs Used for the Treatment of Diabetes. Journal of Clinical Biochemistry and Nutrition, 40, 163-173.

Mohanna, C., \& Nys, Y. (1999). Effect of dietary zinc content and sources on the growth, body zinc deposition and retention, zinc excretion and immune response in chickens. British Poultry Science, 40, 108-114.

Mutabagani, A., \& El-Mahdy, S. (1997). A study of the anti-inflammatory activity of Nigella sativa L. and thymoquinone in rats. Saudi Pharmaceutical Journal, 5, 110-113.

Ouchi, N., Parker, J. L., Lugus, J. J., \& Walsh, K. (2011). Adipokines in inflammation and metabolic disease. Nature reviews. Immunology, 11, 85.

Pavelic, K., \& Hadzija, M. (2003). Medical applications of zeolites. Handbook of Zeolite Science and Technology (pp. 1143-1174). New York: Dekker.

Razavi, B., \& Hosseinzadeh, H. (2014). A review of the effects of Nigella sativa L. and its constituent, thymoquinone, in metabolic syndrome. Journal of Endocrinological Investigation, 37, 1031-1040.

Stenvinkel, P., Ketteler, M., Johnson, R. J., Lindholm, B., ..., \& Girndt, M. (2005). IL-10, IL-6, and TNF- $\alpha$ : Central factors in the altered cytokine network of uremia-the good, the bad, and the ugly. Kidney International, 67, 1216-1233.

Wu, Q. J., Zhou, Y. M., Wu, Y. N., Zhang, L. L., \& Wang, T. (2013). The effects of natural and modified clinoptilolite on intestinal barrier function and immune response to LPS in broiler chickens. Veterinary Immunology and Immunopathology, 153, 70-76.

Yapislar, H., Taskin, E., Ozdas, S., Akin, D., \& Sonmez, E. (2016). Counteraction of apoptotic and inflammatory effects of adriamycin in the liver cell culture by clinopitolite. Biological Trace Element Research, 170, 373-381.

Zaoui, A., Cherrah, Y., Alaoui, K., Mahassine, N., Amarouch, H., \& Hassar, M. (2002). Effects of Nigella sativa fixed oil on blood homeostasis in rat. Journal of Ethnopharmacology, 79, 23-26.

Zaoui, A., Cherrah, Y., Lacaille-Dubois, M., Settaf, A., Amarouch, H., \& Hassar, M. (2000). Effets diurétiques et hypotenseurs de Nigella sativa chez le rat spontanément hypertendu. Thérapie, 55, 379-382.

\section{Copyrights}

Copyright for this article is retained by the author(s), with first publication rights granted to the journal.

This is an open-access article distributed under the terms and conditions of the Creative Commons Attribution license (http://creativecommons.org/licenses/by/4.0/). 